\title{
New Strategies for Controlling the Size, Morphology and Composition of Bimetallic and Trimetallic Catalyst Particles
}

\author{
Christopher J. Kiely ${ }^{1}$, Qian $\mathrm{He}^{1}$, Ramchandra Tiruvalam ${ }^{1}$, Andrew Herzing ${ }^{1}$, Graham J. \\ Hutchings $^{2}$ \\ 1. Department of Materials Science and Engineering, Lehigh University, PA 18015, U.S.A. \\ 2. Cardiff Catalysis Institute, School of Chemistry, Cardiff University, Wales, U.K.
}

Supported bimetallic and trimetallic nanoparticles are being used more frequently nowadays for applications in catalysis. Ideally we would like to be able to synthesize stable alloy nanoparticles with (i) a well-defined and narrow size range, (ii) very specific morphologies (i.e. random alloy, ordered alloy, core-shell structures) and (iii) closely controlled compositions. In reality, our ability to control all of these critical parameters by conventional catalyst preparation methods is still rather lacking. We have found that aberration corrected analytical electron microscopy is an excellent way of performing 'quality control' measurements on such bi- and tri-metallic catalyst systems. In particular, the combination of HAADF imaging and XEDS compositional analysis in the STEM has given us considerable insight into the way in which elemental distributions develop and evolve in these systems during processing. Our studies have especially highlighted the need to create simple synthesis methods which afford a much better control of composition from particle-to-particle in such bi- and trimetallic catalysts.

In this presentation some case studies will be given, using the Au-Pd and Pt-Pd bimetallic and $\mathrm{Au}-\mathrm{Pd}-\mathrm{Pt}$ trimetallic systems as specific examples, to illustrate the complex relationships that can exist between preparation route, compositional homogeneity and catalytic performance.

The most common method of preparing supported Au-Pd bimetallics is via an impregnation route in which $\mathrm{HAuCl}_{4}$ and $\mathrm{PdCl}_{2}$ precursor solutions are co-deposited onto a support followed by drying and calcination. This inevitably generates Au-Pd alloy particles having a broad particle size distribution, with the larger particles being Au-rich and the smaller ones being Pd-rich [1]. Interestingly on oxide supports the $\mathrm{Au}-\mathrm{Pd}$ particles adopt a Pd-rich shell/Au-rich core structure, whereas on activated carbon they remain as random homogenous $\mathrm{Au}-\mathrm{Pd}$ alloys. The sheer structural and compositional complexity of the resulting material often hinders the elucidation of meaningful structure-performance relationships in such impregnated bimetallic catalyst systems.

Colloidal synthesis (or sol-immobilization) methods are becoming more popular because they can deliver much better control over particle size [2]. However, the alloy particles so generated suffer the added complication of being coated with organic ligands and also still exhibit severe compositional inhomogeneities. For example, random alloy Au-Pd, Pt-Pd and Au-Pt-Pd particles prepared in this way always exhibit a systematic size-dependent composition in which the larger particles are $\mathrm{Pd}$-rich and smaller ones are $\mathrm{Pd}$-deficient (i.e. the exact opposite trend to that displayed by analogous materials prepared by impregnation). Sol-immobilization methods also have an apparent advantage in that they can be manipulated (as shown in Figure 1) to allow the deliberate preparation of 'designer' bimetallic morphologies (e.g. Pd-shell/Au-core and Aushell/Pd-core particles). In practice however, we now know that the particle size distribution of the initial 'seed' colloid that forms the core, can lead to drastic variations in shell thickness (and 
hence overall composition) from particle-to-particle. Furthermore secondary nucleation events and thermally induced diffusion effects can also cause further severe deviations away from the expected nominal alloy composition.

To overcome these materials processing limitations, especially over compositional control, we have developed two new synthesis methods for the production of supported bi- and tri-metallic alloys. The first, which we call modified impregnation [3], is a variation on the conventional impregnation technique, in which excess anion ligands are added during the precursor introduction stage in order to better disperse and mix the metal components. This is followed by a simple heat treatment in a reducing atmosphere, to remove any core-shell variants and solely generate the more desirable random alloy morphologies. The second method, known as chemical vapour impregnation (CVI), involves the direct mixing of suitable organometallic precursor compounds with the support material, followed by a thermal decomposition step. The supported alloy materials prepared by both the modified impregnation and CVI routes have a smaller average particle size and significantly improved compositional uniformity from particle-toparticle, which ultimately results in a higher activity and stability, as compared to catalysts prepared via the conventional impregnation or sol-immobilization methods.

References:

[1] A.A. Herzing, M. Watanabe, C.J. Kiely, J.K. Edwards, M. Conte, Z.R. Tang and G.J. Hutchings, Faraday Discussions, 138, (2008), 337-351.

[2] R.C. Tiruvalam, J. Pritchard, N. Dimitratos, J.A. Lopez-Sanchez, J.K. Edwards, A.F. Carley G.J. Hutchings and C.J. Kiely, Faraday Discussions, 152, (2011), 63-86.

[3] M. Sankar, M. Morad, J. Pritchard J, Q. He, J.K. Edwards, S.H. Taylor, A.F. Carley, D.W. Knight, C.J. Kiely and G.J. Hutchings, ACS Nano, 6, (2012), 6600-6613.

[4] The authors wish to thank Drs N. Dimitratos, J.A. Lopez-Sanchez, M. Sankar, M.M. Forde, J.K. Edwards, M. Morad, J. Pritchard and M. Watanabe for their valuable contributions to this work.
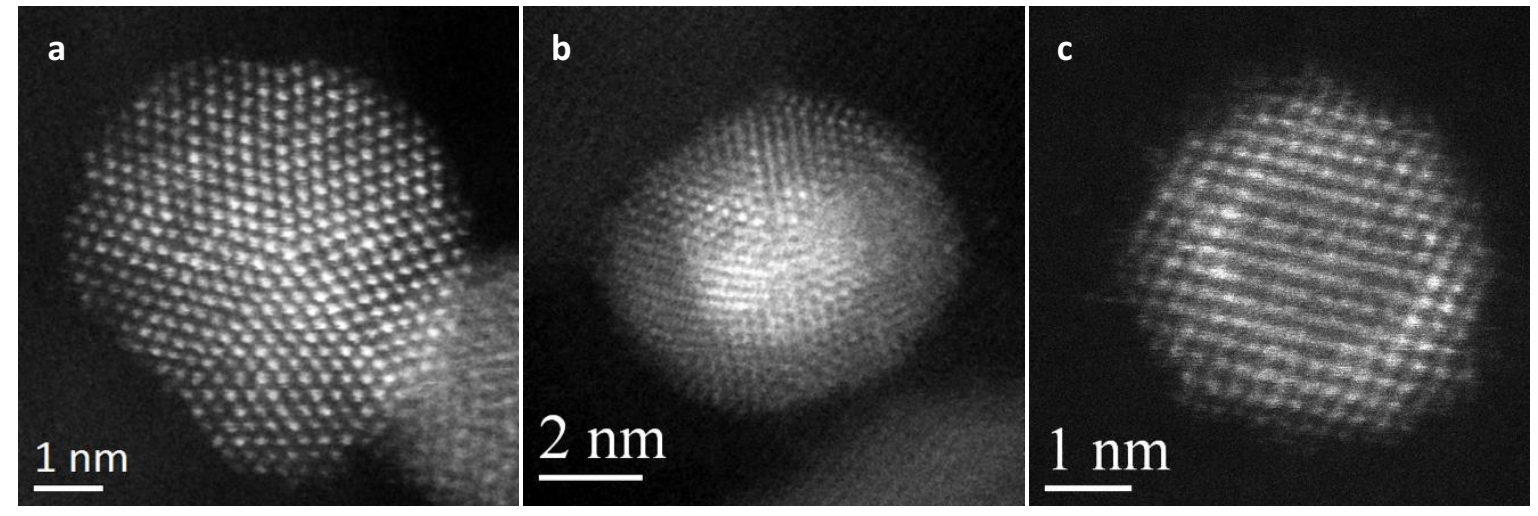

Figure 1. HAADF ( $z$-contrast) images of sol-immobilized Au-Pd catalyst particles: (a) a random alloy $\mathrm{Au}-\mathrm{Pd}$ nanoparticle; (b) a Au-core/Pd-shell nanoparticle, and (c) a Pd-core/Au-shell nanoparticle (from Reference [2]). 